\title{
Corrosion in Control Systems Decrease the Lifetime of the Electronic Devices of the Industrial Plants of Mexicali, BC, Mexico
}

\author{
Gustavo López Badilla1, María Marcela Acosta Gómez , Elizabeth Romero Samaniego², \\ Sandra Luz Toledo Perea ${ }^{2}$, Rodolfo Thomas Guevara Garduño ${ }^{3}$, \\ Carlos Omar Gutiérrez Márquez ${ }^{3}$, Juan Pablo Quezada Hinojosa ${ }^{3}$ \\ ${ }^{1}$ Researcher-Academic, Instituto Tecnológico de Mexicali (ITM), Mexicali, México \\ ${ }^{2}$ Researcher-Academic, Instituto Tecnológico de Ensenada (ITE), Ensenada, México \\ ${ }^{3}$ Students of the Electronic Engineering, Instituto Tecnológico de Mexicali (ITM), Mexicali, México \\ Email: glopezbadilla@yahoo.com
}

Received January 23, 2013; revised March 8, 2013; accepted April 15, 2013

Copyright (C 2013 Gustavo López Badilla et al. This is an open access article distributed under the Creative Commons Attribution License, which permits unrestricted use, distribution, and reproduction in any medium, provided the original work is properly cited.

\begin{abstract}
The principal factor to determine the economical value of the products manufactured in the electronics industry is due to the productive yielding. This is important for the cost of the articles fabricated in this type of industrial plants installed in Mexicali city, where around $80 \%$ of companies are, and which fabricate electronic devices and systems, or have industrial electronic systems and machines to their manufacturing process. Mexicali city is located in the Baja California State of the northwest of Mexico, which is a border city with Calexico in the California State of the United States of America (USA). The region located in Mexicali, is a desert area. Geothermal plant is located in this area, which is an important industry and supplies electricity to this city and its valleys and some cities on southwest of United States for daily activities. This company emits hydrogen sulfide $\left(\mathrm{H}_{2} \mathrm{~S}\right)$ as a main air pollutant that reacts with oxygen in the atmosphere, generating sulfur oxides $\left(\mathrm{SO}_{\mathrm{X}}\right)$. This chemical is dispersed to the city of Mexicali in which industrial plants are located with electronic control systems, and penetrates to indoor rooms. Those cause the corrosion process. The presence of corrosion leads to the deterioration of electrical connectors, the connections of electronic systems and the decreasing of the lifetime of these control systems. Other air pollutants that are considered as chemical agents which cause damage to materials used in the electronics industry, are the sulfurs and nitrogen oxides $\left(\mathrm{NO}_{\mathrm{X}}\right)$, emitted from the traffic vehicle and some industries. This causes the low productive yielding of electrical and electronic devices and systems used in the companies of this city, and is a major concern to specialized people, managers and owners. To analyze the productive yielding of electronic devices and systems installed in indoor of the electronics industry. For this reason, to know the principal causes of it, a study in three industrial plants, to determine the grade level of deterioration of the electronic control systems (ECS) used in the electronics industry of this city was made. The results showed that at major air pollution concentration detected by specialized methods, the lifetime of the ECS was decreased by the generation of corrosion in their electrical connectors and connections. This was caused for the levels of air pollutants mentioned above, than exceed the air quality standards in some periods of the year, added with the levels upper of relative humidity levels (RH) and temperatures of $85 \%$ and $25^{\circ} \mathrm{C}$ in winter and $80 \% 35^{\circ} \mathrm{C}$ in summer, being a main factor of this electrochemical phenomenon.
\end{abstract}

Keywords: Corrosion; Electronic Devices; Control Systems; Industrial Plants

\section{Introduction}

The permanence of an industrial plant in the world marketing depends on their planning production in their manufacturing processes, including the methods of fabrication, and where specialized people are monitoring the productive yielding of the ECS [1]. The ECS are very important in the production activities to control operations of assembly parts of a product, also detecting defects in articles fabricated, and count the partial and total products manufactured. Also the ECS are used in activeties to make risky operations with toxic chemical. The ECS are exposed to atmospheres in indoor of the electronics industry, where in the majorly of times, if the 
companies do not have control of the concentration levels of sulfurs principally, RH levels and temperature, can originate aggressive environments in some areas of indoor of the industrial plants and cause the corrosion process. In the majorly of the electronics industry, the indoor atmospheres are controlled, especially in clean rooms, but chemical agents derived of the sulfur, are gaseous and penetrated by small holes, roof of buildings and air conditioning systems. The control of indoor environments at the maximum scale and not as a total percentage, promotes the clean atmospheres to avoid the deterioration of the articles fabricated and the ECS, to have the maximum productive yielding. All factors of the functions of ECS are necessarily verified in some periods of the day, weekly, monthly and seasonally; to be sure that these electronic equipments are operating correctly. The presence of corrosion in indoor of the electronics industry, decreases the productive yielding and the low value of the article fabricated, derived by the capacity of the ECS in the industrial process. The damage of the ECS can decrease their functions and reduce their electrical properties, decreasing their lifetime and causing economical losses. This is affected in the major times, by the uncontrolled microclimate of indoor in the industrial plants, causing the presence of chemical processes as the corrosion that affects the operation of the ECS [2]. Air pollutants that are in indoors of the electronics industry, are deposited in the electrical connections and connectors, and with the levels of $\mathrm{RH}$ and temperature mentioned above, promotes very easy and quickly, the electrochemical phenomena. The corrosion that occurs in Industrial plants generates great economic losses, and various research institutions works a lot in relation with companies, about these cases with different methods to know the origin of the different types of corrosion that can arise. The two more principal types of corrosion which occur in the companies located in Mexicali, are the uniform and pitting corrosion, where the first type of corrosion is detected very easy because appears in the majorly of the metallic surface and occurs frequently in the winter season. The pitting corrosion which appears princepally in the summer months, is more difficult to detect, because in this type are formed corrosion products over the pitting. For this reason can run at low or fast velocity into the metal and not outside of this, causing the deterioration of the metals of the electrical connectors and connections and therefore the electrical failures [3].

\subsection{The Global Electronics Industry}

The world marketing competition, has led to develop technological advances, particularly in the area of the electronics, which are increasingly being manufactured microelectronic components, very robust and with a greater number of operations. The tendency to miniatu- rization of the technology is a leader in the development of equipment electronics such as cell phones, computers, televisions and a lot of electronic devices. Materials used in the electronic components are alloys based especially on aluminum (integrated circuits with at micro and nano size of wire), copper contacts nickel electroplated with gold to improve the resistance to corrosion, but increase the cost of this [4]. The characteristics of modern electronic equipment with high voltage, contemplate three principal factors as speed of operations, very small electrical currents and the miniaturization and extremely sensitive to corrosive agents. This can lead to some electrical failures in the microelectronic components, generating technical problems in the ECS, caused by the atmospheric pollutants and the variations of humidity and temperature. It attacks the principal metallic materials mentioned above, used in the electronic devices. The microchips are protected by specialized coatings to prevent that not suffer any type of damage immediately by the exposure to aggressive environments and climate changes decreasing their functionality and lifetime. The

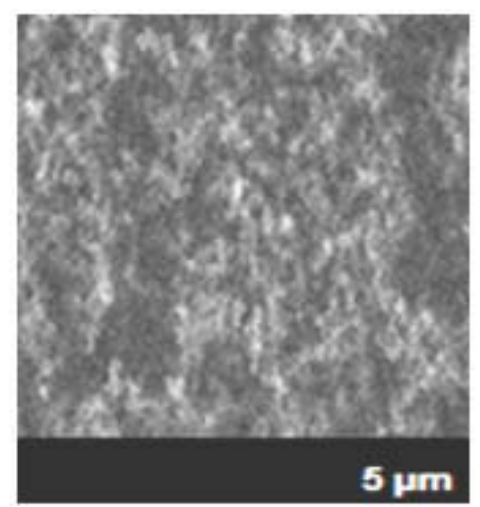

(a)

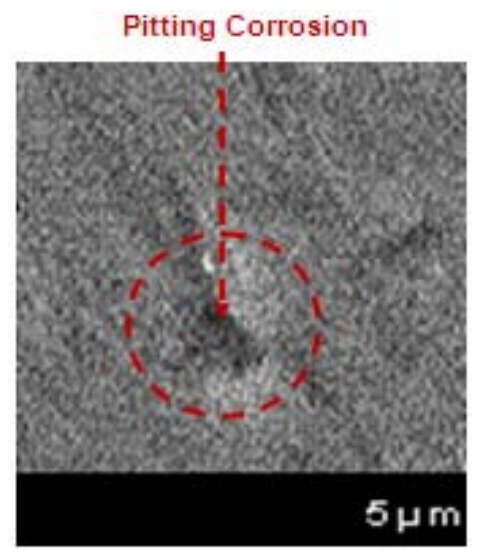

(b)

Figure 1. Microphotographs of metallic surface deteriorate of electrical connections of ECS caused by (a) uniform corrosion and (b) uniform and pitting corrosion in an industrial plant in Mexicali (2011). 
corrosion damage tolerance in electronic components is of very small order of magnitude at 10 to12 nano grams. According to the research studies, the order of the width of the films of electronic boards is of 50 microns, and in hybrid circuits (HC), the space should be of 2.5 microns. This type of microelectronic devices corroded is shown in Figure 1.

As shown in Figure 1, the metallic surfaces of the microelectronic devices were damaged by the corrosion process, indicating in each microphotography the presence of uniform corrosion, but in the section b, appeared pitting corrosion with a little pit where not was observed to the naked eye, and only was detected with the microanalysiswith the Scanning Electron Microscopy (SEM) [5].

\subsection{Corrosion in the Electronics Industry}

The electronic boards suffer a variety of problems in their electrical conduction surfaces by the presence of the air pollutants as sulfurs principally and the levels of $\mathrm{RH}$ and temperature. When its parameters are combined, the atmospheric agents react very easy and quickly with the metallic surfaces, decreasing the resistance to the corrosion of the connectors and electrical conductive paths. This causes the formation of metallic filaments that grow between routes where not debit growth, causing electrical conductivity between terminals metal (pins), or metallic unions [6]. The conditions required for this type generating a combination of ionic contamination, are levels of $\mathrm{RH}$ and temperatures less than $70 \%$ and $30^{\circ} \mathrm{C}$, especially in clean rooms of the electronics industry, with lower levels, and depending of the voltage values of application of the electrical sources. The ECS used in very dry conditions almost not suffer from the corrosion, but at high $\mathrm{RH}$ and low temperatures in some months of winter, and high $\mathrm{RH}$ and temperatures in the summer months temperatures, originates the condensation process, forming visible or invisible thin films of water on the surface of the metallic connectors and connections, beginning the corrosion. This originates the absorption very fast of the air pollution by the micro and macro electronic components, decreasing the metallic surfaces by the deterioration and for consequence the strength of the material forming metallic dendrites (Figure 2).

As shown in Figure 2, in the both microphotographs (a and b), the pathways, where flow the electrical current by the corroded areas, indicating that corrosion products are the wrong pathways in the metallic surface and causes bad electrical function in the ECS.

\subsection{Atmospheric Corrosion}

The climatic factors generate the dispersion of air pollutants such as fine particles and gases: hydrogen sulfide,

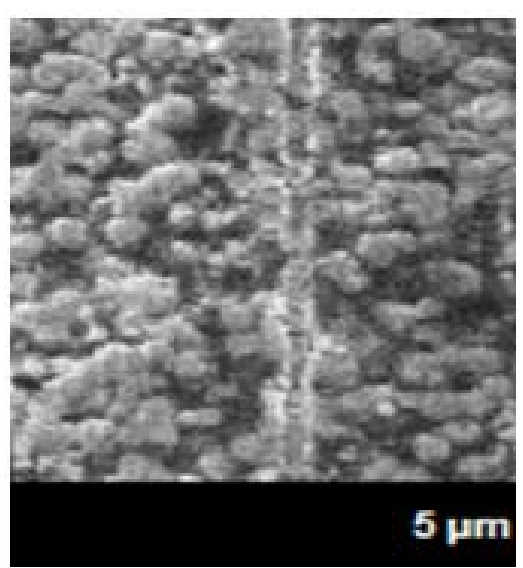

(a)

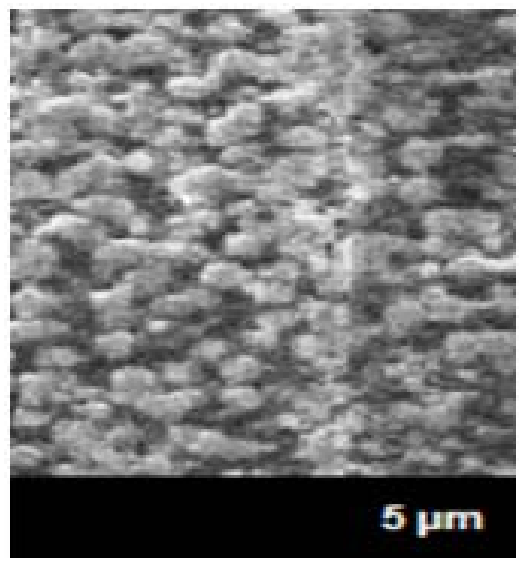

(b)

Figure 2. Microphotography of metallic dendrites formed in pathways of electronic devices in (a) summer and (b) winter.

sulfur dioxide and nitrogen oxides principally. These air pollutants, sometimes exceed the standards of air quality in this region. These contaminants are detected by specialized teams of Environmental Monitoring Stations (EMS) installed in strategic places of the Mexicali city. These chemical agents are emitted by external sources such as traffic, industrial plants, geothermal fields, soil erosion and microorganisms, which penetrate to the indoor of the electronics industry. At levels higher than $75 \% 25^{\circ} \mathrm{C}$ of $\mathrm{RH}$ and temperature, that is common in some periods of the year in Mexicali, are obtained the time of wetness (TOW), indicating the periods in which the metallic surfaces of microelectronic devices kept moist for at least one day, causing the electrochemical corrosion process. The pollution levels are evaluated according to standard regulations that indicate the concentration levels regulated by environmental institutions in each country. In Mexico, the Secretaria del Medio Ambiente y Recursos Naturales (SEMARNAT), in mutual agreement with the Environmental Protection Agency (EPA) of USA [3,7], are responsible for the 
regulation of the pollution emissions of the outdoor and indoor of the industrial plants. With international agreements, on the border of Mexico and the United States, SEMARNAT and the EPA, have installed Environmental Monitoring Stations (EMS) and Meteorological Monitoring (MMS). With thus systems are obtained information of outdoor pollution that affects indoor atmospheres of the electronic industry, at high levels of air pollution and with drastic variations of RH and temperature, showing diverse values in different seasonal periods. The chemical agents, which are monitored by the EMS in this city, are $\mathrm{H}_{2} \mathrm{~S}$ from the geothermic plant, sulfurs dioxide $\left(\mathrm{SO}_{2}\right)$, carbon monoxide (CO), nitrogen oxides $\left(\mathrm{NO}_{\mathrm{X}}\right)$, ozone $\left(\mathrm{O}_{3}\right)$ and particulate matter $\left(\mathrm{PM}_{10}\right.$ and $\mathrm{PM}_{2.5}$ ), which are reported by the National Ambient Air Quality Standards (NAAQS, 2006). Additionally are monitored the volatile organic compounds (VOC) generated in indoors of the companies by specialized techniques. Also this monitored ammonia that is inorganic agent and has an effect on the deterioration of metallic connectors and connections of the ECS, and even at low concentrations, have a significant effect on the corrosion phenomenon [2].

\subsection{Corrosion Types in the Electronics Industry}

According to the humidity originated in indoors of industrial plants, material of electronic devices generates different corrosion processes, such as those explained below [1]:

A) Corrosion voltaic. This type of corrosion is generated in a small space between electronic circuit components, when a voltage is applied to a device, generating the voltage gradients in order of micro-volts in electrical connectors and connections, resulting in accelerated electrochemical corrosion reactions and ion migration. The integrated circuits are very susceptible to corrosion. The combination of electrical fields, with atmospheric humidity and air pollution are the main promoters of this type of corrosion in metals used in the microelectronic devices. The corrosion can occur only with the presence of low humidity in sometimes. The $\mathrm{pH}$ generated by a cathodic reaction is for the reduction of water. High $\mathrm{pH}$ levels result in a solution in the passive film, with a surface of the metal oxide and aluminum substrates and increases the electrical resistance, generating an open circuit.

B) Electrolytic metal migration. In presence of moisture and an electric field, silver ions migrate to a charged surface cathodically, forming dendrites, which grow as bridges between contacts, possibly causing short circuits to reduce the production yielding of the ECS and decreasing their life time, and in sometimes causing fires. Always a small volume of the dissolved metal forms the dendrites as a large pathway. Other materials which are susceptible to metal migration ions are the gold, tin, lead, palladium and copper. Dendrites can be of silver, copper, tin, lead or combination of these metals and cause electrical failures in the microelectronic devices and also to ECS, by short circuits. The dendrite is generated and grows very fast between electrical connectors and connections originating electrical failures in the ECS. The growth rate of dendrite depends on the applied voltage and the amount of contamination atmospheric combined with the moisture, which affect the metal surface.

C) Formation of pores and cracks in electrical contacts and connections metal. To prevent the connectors and electrical contacts stained by the corrosion process in metals such as gold, the process is performed on the surface of silver contacts and connectors. However, defects in protective coatings of metals can expose and corrode the substrate material and create imperfections like pores and cracks. If the substrate is copper or silver, and it is exposed to environments with sulfates or chlorides, the products corrosion, cracks will generate pores, and if gold added to silver forms a high resistance in the conductive layer, whereby open circuits are generated.

D) Fretting corrosion on separate jacks with fine finishes. It results in the formation of tin oxides boards electronic or electrical contacts. The problem starts very often when the tin is used or replaced by gold metal, being more economical. The possible solution is to replace the hand or use a little more expensive metals.

E) Galvanic corrosion. It occurs when two different metals, such as aluminum and gold come together, as it is in the encapsulation of integrated circuits. The polymers used are packaging as a pair porous and plastic tapes that seal metal joints are manufactured as electronic devices ceramic or metallic. Sometimes, humid environments generate galvanic corrosion conditions.

F) Corrosion in industrial processes. Integrated circuits are environments exposed to numerous aggressive attack ionic or wet and aluminum, which are the main compounds. The ion etching requires a combination of gas and if this is formed as chlorides aluminum covering the metallic structures of the ECS, which are acidic agent and generates acid moisture. The ionic contamination occurs in soldering processes and handling of materials used to fabricate microelectronic devices with very thin films, originated by dust and changes climate.

G) Microcorrosion in the manufacture of integrated circuits. The metallization of aluminum and copper alloys can form inter-metallic compounds like $\mathrm{Al}_{2} \mathrm{Cu}$ with a large grain boundaries. This is the beginning of the dissolution of metals which form a micro-pitting during the etching.

H) Corrosion by chlorinated solvents or halogenated. Liquid solvents steam or used in the manufacture of integrated circuits, are mainly the factors which corrode 
aluminum components. Contaminated water of solvents increases the time of the presence of corrosion and also increases the corrosion rate, which is the speed at a metal which disintegrates. The stabilized solvent dissolution with alcohol or aromatic solvents is the main cause of halogenated solvents breaking and forming chlorine ions, corroding the aluminum and copper alloys, with major effect in the aluminum.

I) Corrosion in welding. Corrosion resistance joints of tin and lead in aqueous and gaseous environments is a function of the alloy. This significantly improves when increases more than 2 times the rate of the alloy. Lead forms unstable oxide, which reacts readily with chlorides, borates and sulfates.

\subsection{Mexicali as Arid Region}

The topography of the Mexicali city is important to the dispersion of the air pollutants mentioned above, penetrating to indoor of the electronics industry where there are about 156 industrial plants according to the Asociación de Maquiladoras de Mexicali (AMAQ) [8]. These contaminants in certain seasonal periods generate aggressive climates mainly in the production areas and warehouses of indoor of these industrial plants, where are used and store the microelectronic devices and the ECS. The climate is an important aspect, and in the operational functions of the ECS of this city. The RH in some periods of the year, of this city is around from 50\% to $90 \%$ and the temperature ranges are near of $0^{\circ} \mathrm{C}$ and the higher value is around $45^{\circ} \mathrm{C}$. These climate changes vary the outdoors, and also the indoors of the industrial plants, contributing to the deterioration of materials $[9,10]$. Dust and chemicals in indoor environments, are added to the microelectronic components of ECS such as computers, measuring instruments and industrial machinery as shown in Figure 3.

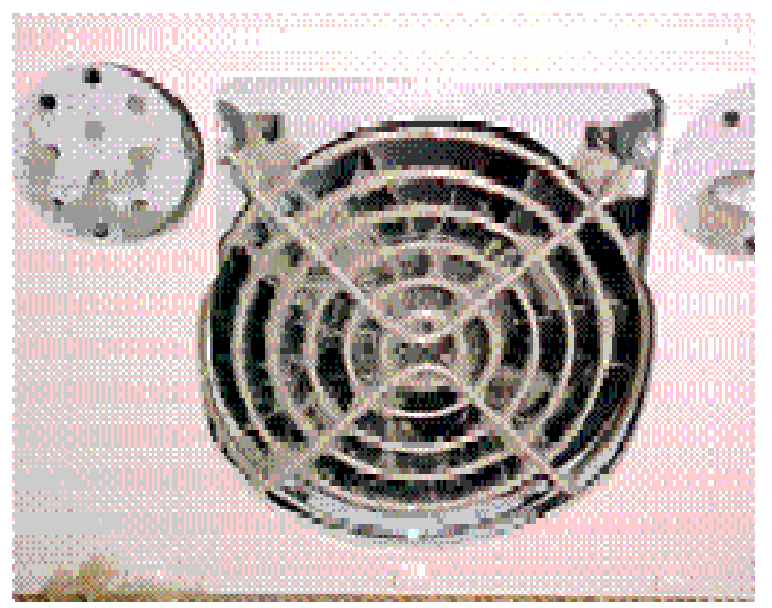

Figure 3. Electric fan controlled by ECS of an industrial machine contaminated by dust and chemical agents.

\subsection{Mathematical Simulation}

MATLAB is software called Matrix Laboratory, being mathematical programming that offers integrated development environment (IDE) with proprietary operations of language called language $\mathrm{M}$. It has basic features as array manipulation, data representation and functions, implementation of algorithms, creation of user interfaces as GUI and communication with programs in other languages and other hardware devices [11]. The MATLAB package has two additional tools that expand their services: Simulink and Guide Commands. In this section, the software was used to make correlations of climatic factors and air pollutants related to the corrosion rate (CR), which represents the deterioration levels of the materials used in the electrical connectors and connections of the ECS.

\section{Methodology}

Humidity and temperature are the two most important climatic parameters related to the origination of the corrosion process. For this study was used information of the air pollutants mentioned above, and also the temperature and RH of 2010 to 2011 obtained of the specialized equipments and the EMS. Based on the statistical information of the climate factors was made an analysis of the generation of the different types of corrosion in the ECS, which use microelectronic devices. According to the emission supplies, the levels of air pollution are concentrated in some areas of Mexicali, principally where are located the electronics industry. In Mexicali the temperature is very hot in summer until major of $45^{\circ} \mathrm{C}$ in some periods of this season and very cold nights until near of $0^{\circ} \mathrm{C}$ in the winter. The maximum and minimum recorded monthly periods of $\mathrm{RH}$ and temperature of indoors of industrial plants, showed ranges varying under $10 \%$ and above $90 \%$ for $\mathrm{RH}$ in summer and winter, and temperatures in the winter of $0^{\circ} \mathrm{C}$ to nearly $50^{\circ} \mathrm{C}$ in the months of June to August. The TOW monthly values correspond to low and medium levels of corrosivity, with ISO 9223 and ISO 11844-1 [12-14]. The study was made in three industrial plants of this city principally of the electronics area from 2010 to 2011.

\section{Results}

The levels of $\mathrm{RH}$ and temperature promote the process and influence the dispersion of air pollutant from natural and anthropogenic sources, causing the corrosion process. In the summer the atmospheric pollution, is based on the temperatures in the range from $30^{\circ} \mathrm{C}$ to $42^{\circ} \mathrm{C}$ and the $\mathrm{RH}$ levels ranging from $55 \%$ to $85 \%$ with a higher intensity is observed of $90 \%$, representing high dispersion of air pollutants and in winter at temperatures near of $0^{\circ} \mathrm{C}$ are presented low dispersion, remaining near of the industrial 
plants and penetrating to its companies. This originates in some periods of the year and some areas, a high concentration of pollutants, increasing the CR and the deterioration of the metallic surfaces of ECS.

\subsection{Correlation Analysis of CR in ECS and Climatic and Environmental Factors}

The CR was influenced by the exposition at different levels of the air pollutants, damaging the microelectronic devices. In this study a correlation analysis was made to know the grade of deterioration of the micro components of the ECS. At different levels of RH, temperature and concentration levels of air pollution, were obtained diverse values of correlation, indicating the major indices in winter where occur the condensation phenomenon and the fast and easy adherence of the air pollutants, as shown in Table 1. The decreasing of the ECS in sometimes for the corrosion process generates low productive yielding and every time the microelectronic devices suffer damage decreasing their lifetime. The correlation was divided into four ranges.

Table 1 shows the levels in each seasonal period where are correlated the climatic factors with the CR. In the spring season, the major CR was 0.79 at the ranges from $36^{\circ} \mathrm{C}$ to $45^{\circ} \mathrm{C}$ and 0.78 from $76 \%$ to $90 \%$. In this season, the presence of corrosion was low, without high effects in the deterioration of metallic surfaces and the lifetime of ECS. The lower value of CR was 0.76 at levels from $0{ }^{\circ} \mathrm{C}$ to 0.78 from $0 \%$ to $40 \%$. In summer the higher value of CR was 0.84 at levels from $36^{\circ} \mathrm{C}$ to $45^{\circ} \mathrm{C}$, where was presented pitting corrosion at value higher than $75 \%$ and $35^{\circ} \mathrm{C}$, and the CR of 0.85 was the highest value at ranges from $76 \%$ to $90 \%$. In autumn was indicated the lowest value of the analysis with 0.75 from $0^{\circ} \mathrm{C}$ to $20^{\circ} \mathrm{C}$ and the higher level was intensity 0.77 of $\mathrm{CR}$ at ranges of $21^{\circ} \mathrm{C}$ to $35^{\circ} \mathrm{C}$. In this season the corrosion appears at low intensity as same as the spring season. In winter the $\mathrm{CR}$ was represented the highest value with 0.90 at ranges from $21^{\circ} \mathrm{C}$ to $35^{\circ} \mathrm{C}$ and 0.90 at levels from $41 \%$ to $75 \%$. In this season was presented uniform corrosion at values higher of $70 \%$ and $30^{\circ} \mathrm{C}$. This represented that in winter the metals suffer of more damage than in other periods of the year.

\subsection{Mat Lab Simulation}

A factor shown in this study was that at low and medium concentrations of sulfurs, where the CR was very fast, but at high concentration, the $\mathrm{CR}$ was low, as mention the standard concentration level of $75 \mathrm{ppb}$ according to the EPA. Furthermore, in winter, levels near of $10^{\circ} \mathrm{C}$ and ranges of $\mathrm{RH}$ from $35 \%$ to $70 \%$, there was a lower incidence of condensation of water on the metal surface and the CR was high. In the winter time, it has higher air pollution in the temperature range of $2^{\circ} \mathrm{C}$ to $13^{\circ} \mathrm{C}$ and $\mathrm{RH}$ levels from $34 \%$ to $70 \%$. At values of temperature from $15^{\circ} \mathrm{C}$ to $20^{\circ} \mathrm{C}$ and $\mathrm{RH}$ of $45 \%, 75 \%$, the $\mathrm{CR}$ was higher. At temperatures of $25^{\circ} \mathrm{C}$ to $30^{\circ} \mathrm{C}$, with $\mathrm{RH}$ levels from $30 \%$ to $75 \%$, there is a low dispersion of air pollutants, showing a CR high and more impact in the deterioration of the metallic surfaces of ECS. The analysis in this research in the summer for the dispersion of air pollutants, indicating that focus on larger scale, at temperatures below $20^{\circ} \mathrm{C}$, showing a CR low at $35 \%$ to $55 \%$, being a high dispersion of the air pollutants in indoor of the industrial plants located in Mexicali. The corrosion was stabilized at $40^{\circ} \mathrm{C}$ and was a $\mathrm{CR}$ very low as shown in Figures 4 and 5.

In Figure 4, the maximum $\mathrm{CR}$ of the correlation was $391 \mathrm{mg} \cdot \mathrm{m}^{2} /$ year at $28^{\circ} \mathrm{C}$ and $78 \%$ temperature, and $\mathrm{RH}$ levels showed fast deterioration of the metallic surfaces of electrical connectors and connections of the ECS. This causes lack of electrical current and not functions of ECS, originating damage in some microelectronic components and decreasing their lifetime. The minimum CR was 9 $\mathrm{mg} \cdot \mathrm{m}^{2} /$ year at $11^{\circ} \mathrm{C}$ and $54 \%$ temperature and $\mathrm{RH}$ levels. In Figure 5, the maximum CR was $209 \mathrm{mg} \cdot \mathrm{m}^{2} /$ year at

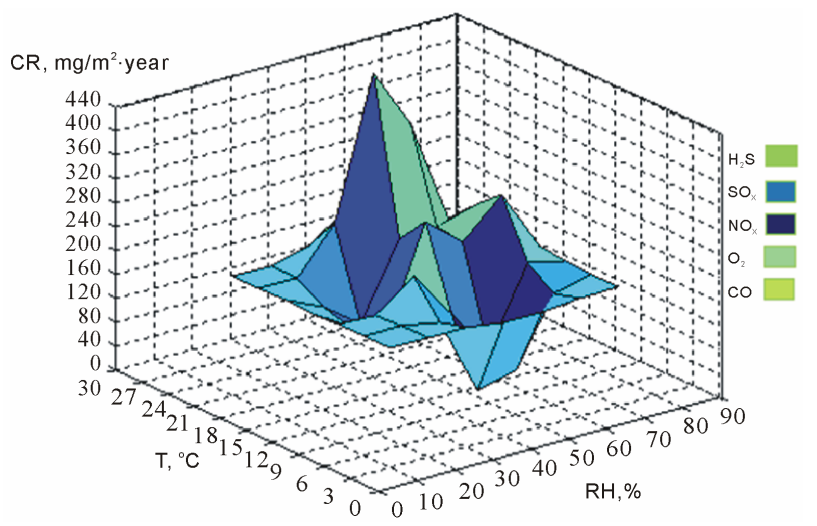

Figure 4. Correlation of CR with climatic factors and sulfurs in Mexicali in winter (2010-2011).

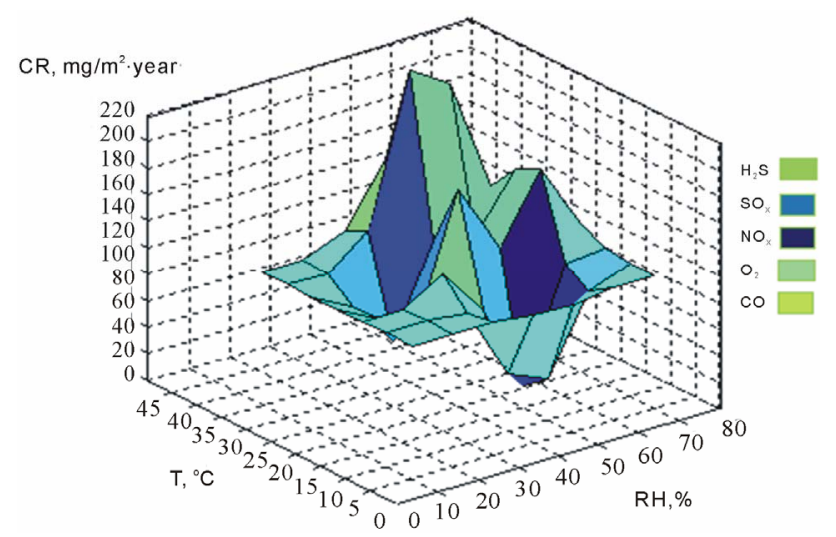

Figure 5. Correlation of CR with climatic factors and sulfurs in Mexicali in winter (2010-2011). 
Table 1. Correlation analysis of CR in different seasonal of the year.

\begin{tabular}{ccccccc}
\hline & \multicolumn{2}{c}{ Ranges } & \multicolumn{2}{c}{ Ranges } & \multicolumn{2}{c}{ Ranges } \\
\cline { 2 - 7 } & $\mathrm{T}, 0^{\circ} \mathrm{C}-20^{\circ} \mathrm{C}$ & $\mathrm{RH}, 0 \%-40 \%$ & $\mathrm{~T}, 21^{\circ} \mathrm{C}-35^{\circ} \mathrm{C}$ & $\mathrm{RH}, 41 \%-75 \%$ & $\mathrm{~T}, 36^{\circ} \mathrm{C}-45^{\circ} \mathrm{C}$ & $\mathrm{RH}, 76 \%-90 \%$ \\
\hline Spring & 0.76 & 0.78 & 0.77 & 0.79 & 0.79 & 0.78 \\
Summer & 0.82 & 0.83 & 0.84 & 0.86 & 0.84 & 0.85 \\
Autumn & 0.75 & 0.74 & 0.77 & 0.75 & 0.76 & 0.77 \\
Winter & 0.89 & 0.87 & 0.90 & 0.90 & 0.88 & 0.89 \\
\hline
\end{tabular}

$42^{\circ} \mathrm{C}$ and $78 \%$ of temperature, and $\mathrm{RH}$ levels and the minimum value of $\mathrm{CR}$ was $6 \mathrm{mg} \cdot \mathrm{m}^{2} /$ year at $19^{\circ} \mathrm{C}$ and $58 \%$ of temperature and $\mathrm{RH}$ levels.

\section{Conclusion}

With increasing miniaturization of systems electronics and the explosive increase in its availability, it is estimated that the corrosion and deterioration of metal materials electronic will increase causing incalculable conesquences. The corrosion phenomena have an effect on the operation of ECS with microelectronic used in the electronics industry. The presence of acidic substances in indoor of industrial plants to damage the electrical connectors and connections of ECS, originates aggressive environments, which generates very fast the deterioration and decreases the lifetime of the ECS of industrial equipments and machines. This affects the functionability every time and originates electrical unoperation that causes the defect before they present their standard lifetime, and begins to fail electrically. This concerns the specialized personas and managers because it generates unnecessary costs. The types of corrosion detected in the connections of electronic devices as microchips were the uniform and pitting corrosion, which are generated by the different values of humidity and temperature in the diverse periods of the year. The climatic factor with major effect was the humidity, which was changing in according to the seasonal period. The uniform corrosion was formed in the winter season and the pitting corrosion appeared more frequently in the summer period. This was evaluated to determine the periods of the year, which are a corrosion rate (CR) high in some periods of the year, decreasing the manufacturing process until $70 \%$.

\section{REFERENCES}

[1] L. B. Gustavo, "Caracterización de la Corrosión en Materiales Metálicos de la Industria Electrónica en Mexicali,” PhD Thesis, University of Baja Califormia in Mexicali, 2008, 115 p.

[2] B. G. Lopez, S. B. Valdez, K. R. Zlatev, P. J. Flores, B. M. Carrillo and W.M. Schorr, "Corrosion of Metals at
Indoor Conditions in the Electronics Manufacturing Industry," Anti-Corrosion Methods and Materials, Vol. 54, No. 6, 2007, pp. 354-359.

[3] "Metals Tests Methods and Analytical Procedures," Annual Book of ASTM Standards (G4, G15, G31), Section 3, Vol. 3, No. 1, 2001.

[4] American Society of Heating, Refrigerating and AirConditioning Engineers, “ASHRAE Handbook: Heating, Ventilating, and Air-Conditioning, Applications," American Society of Heating, Refrigerating and Air-Conditioning Engineers Inc., 1999.

[5] A. Nishikata and Y. Ichihara, "The Effect of Time of Wetness (TOW) in Metallic Components," Corrosion Science, Vol. 37, No. 37, 1995, pp. 134-138.

[6] L. Velasco, "La Contaminación Atmosférica de las Grandes Urbes,” Ciencia y Desarrollo, Marzo-Abril, 1996.

[7] NAAQS-National Ambient Air quality Standards, US, "Environmental Protection Agency (EPA)," Consultado en. http://www.epa.gov/air/criteria.html

[8] Asociación de Maquiladoras de Mexicali-AMAQ, Departamento de Estadística, Reporte Anual de la Industria en Mexicali, Gobierno Municipal, 2008.

[9] D. Camuffo and A. Bernardi, "Controlling the Microclimate and the Particulate Matter inside the Historic Anatomic Theatre, Padova,” Museum Management and Curatorship, 1997, pp. 285-298.

[10] M. Morcillo, "Evaluación de la Polución Ambiental en Interiores de Empresas, Madrid, España,” Revista Iberoamericana de Corrosión, Vol. 42, No. 17, 1996, pp. 112 115 .

[11] B. H. Duncan, "Walsh an Engineer's Guide to MATLAB, 2e: With Applications Electrical Systems,” Prentice Hall, Upper Saddle River, 2005.

[12] ISO 9223, "Corrosion of Metals and Alloys, Corrosivity of Atmospheres, Classification,” 1992.

[13] ISO 11844, “Corrosion of Metals and Alloys, Classification of Low Corrosivity Indoor Atmospheres. Part 1: Determination and Estimation of Indoor Corrosivity,” 2006.

[14] ISO 11844, "Corrosion of Metals and Alloys, Classification of Low Corrosivity of Indoor Atmospheres. Part 2: Determination and Estimation Attack in Indoor Atmospheres," 2005. 